\title{
Effect of Polarization Mode Dispersion on a Coherent Optical System with Pilot Carrier
}

\author{
Ming-Seng Kao and Jinshown Wu
}

\begin{abstract}
The effect of fiber polarization mode dispersion (PMD) on a coherent optical system with pilot carrier is twofold. First, the PMD causes state of polarization (SOP) mismatch between the message signal and pilot carrier. Second, the degrees of polarization (DOP) of the message signal and pilot carrier are degraded by PMD. Because coherent reception relies both on the SOP's and DOP's of the waves, system performance is degraded by PMD. The effect of SOP mismatch causes intermediate frequency (IF) signal amplitude degradation whereas DOP variation not only results in IF signal amplitude degradation but further induces IF noises. Here we derive analytical expressions to evaluate the performance degradation. It is shown that there is no performance degradation if only one principle state of polarization is excited and maximum degradation occurs when both principle states of polarization are equally excited. We also find that significant degradation may happen when large differential group delay exists.
\end{abstract}

\section{INTRODUCTION}

C OHERENT optical communications attracted worldwide research interests in the past decade. The increased receiver sensitivity and tuning capability are two salient features of coherent systems over intensity modulation systems. However, because of the need of a local oscillator (LO) whose SOP must match that of the incoming message signal, a coherent receiver is inevitably complicated and is difficult to implement. Approaches to release this difficulty has been proposed. For example, we can use the carrier amplification technique to eliminate the need of LO laser, [1], [2]. By selectively amplifying the carrier at the receiving end, the carrier can play the same role as an LO so that simple reception is achievable. On the other hand, it is realized that a highpower LO laser indeed can be shared by many receivers and therefore it can be placed at a distribution center [3] or at a remote hub [4] and distributed with the message signal to many receivers. As the LO carrier already presents in the distribution fiber, the LO laser and its associated circuitry in an usual coherent receiver are eliminated and direct detection receiver can be used to receive coherent signal. In the above coherent systems, the LO signal is not locally generated at the receiver but coming with the message signal through the distribution fiber. The LO signal now behaves like a pilot carrier associated with the message signal which eases coherent detection.

Manuscript received July 19, 1991; revised May 20, 1992

M. Kao is with the Department of Communication Engineering, National Chiao Tung University, Hsinchu, Taiwan, Republic of China.

J. Wu is with the Department of Electrical Engineering, National Taiwan University, Taipei, Taiwan, Republic of China.

IEEE Log Number 9203413
An ordinary single-mode optical fiber is actually a twomode fiber because two orthogonally polarized eigenmodes can simultaneously propagate. Usually the two eigenmodes do have different propagation constants due to imperfection in fiber manufacture and deployment which results in PMD Therefore, a linearly polarized wave launched at the fiber input will in general become elliptically polarized at the output because of PMD [5]. Also the DOP of a lightwave, defined as the ratio of the intensity of the completely polarized component to the total intensity, will be affected by PMD as the wave propagates along a fiber [6]. Thus the PMD changes both SOP and DOP of a propagating wave.

In a coherent optical system with a pilot carrier, for example the LO pilot carrier mentioned above, we match the SOP of the pilot carrier with that of the message signal at the transmitting end and distribute them through single-mode fiber to receivers. Because of PMD in the distribution fiber, the SOP's and DOP's of the received message signal and pilot carrier are changed. Because coherent reception relies on both SOP's and DOP's of the message signal and pilot carrier, system performance is expected to be degraded by PMD. A worst-case study which considered the degradation due to SOP only was carried out in [7]. Here a complete treatment of the problem is performed by considering both the effects of SOP and DOP and general results are provided to evaluate the performance degradation.

\section{ANALYSIS}

To study the effect of PMD on system performance, we adopt the formulation of principal states of polarization (PSP's)[5]. The model provides a simple way to describe PMD without knowing the details along the fiber which is useful in practical applications. It is shown that there exists two orthogonal input PSP's whose corresponding output SOP's are invariant with frequency to first-order only if there is negligible polarization-dependent loss. In the following the effect of SOP is studied first, then we consider the effect of DOP degradation, and finally both effects are combined to evaluate the total impact of PMD on system performance.

\section{A. Effect of $S O P$}

An incident optical wave in a fiber will in general excite two PSP's and its optical power is carried by the two PSP's. Let $\vec{E}_{s}$ and $\vec{E}_{c}$ denote the fields of the message signal and the pilot carrier, respectively, which are linearly polarized along the same direction and are incident on a single-mode fiber. In general, the waves will each excite two PSP's and their 
optical powers are carried by the PSP's. Let the two PSP's be specified by two unit vectors $\hat{a}_{+}$and $\hat{a}_{-}$, respectively, then $\vec{E}_{s}$ and $\vec{E}_{c}$ can be expressed as

$$
\begin{aligned}
\vec{E}_{s}(t)= & A_{s} e^{j\left(w_{s} t+\phi_{s}\right)} \\
& \cdot\left[c_{+} e^{j m(t)} \hat{a}_{+}\left(w_{s}\right)+c_{-} e^{j m(t)} \hat{a}_{-}\left(w_{s}\right)\right] \\
\vec{E}_{c}(t)= & A_{c} e^{j\left(w_{c} t+\phi_{c}\right)}\left[c_{+} \hat{a}_{+}\left(w_{c}\right)+c_{-} \hat{a}_{-}\left(w_{c}\right)\right]
\end{aligned}
$$

where $A_{s, c}, w_{s, c}$, and $\phi_{s, c}$ denote the amplitudes, angular frequencies, and phases of the two waves, respectively. Here the phase information $m(t)$ is carried by the two PSP's of $\vec{E}_{s} . c_{ \pm}$stand for the expansion coefficients along the two PSP's. Since the two waves are linearly polarized along the same direction, we assume that they have the same expansion coefficients along the two PSP's. We further normalize $c_{ \pm}$ such that

$$
c_{+}^{2}+c_{-}^{2}=1 \text {. }
$$

With negligible polarization-dependent loss, the received fields can be formulated as [9]

$$
\begin{aligned}
\vec{E}_{s}(t)= & A_{s} e^{-\alpha \frac{L}{2}} e^{j\left(w_{s} t+\phi_{s}\right)} \\
& \cdot\left[c_{+} e^{j\left[m(t)+\psi_{+}\left(w_{s}\right)\right]} \hat{b}_{+}\left(w_{s}\right)\right. \\
& \left.+c_{-} e^{j\left[m\left(t-\tau_{p}\right)+\psi_{-}\left(w_{s}\right)\right]} \hat{b}_{-}\left(w_{s}\right)\right] \\
\vec{E}_{c}(t)= & A_{c} e^{-\alpha \frac{L}{2}} e^{j\left(w_{c} t+\phi_{c}\right)} \\
& \cdot\left[c_{+} e^{j \psi_{+}\left(w_{c}\right)} \hat{b}_{+}\left(w_{c}\right)+c_{-} e^{j \psi_{-}\left(w_{c}\right)} \hat{b}_{-}\left(w_{c}\right)\right]
\end{aligned}
$$

where $\alpha$ is the fiber loss, $L$ is the length, and $\tau_{p}$ is the differential group delay between the two PSP's. $b_{ \pm}\left(w_{s, c}\right)$ denote the corresponding output SOP's of $\hat{a}_{ \pm}\left(w_{s, c}\right)$ whereas $\psi_{ \pm}\left(w_{s, c}\right)$ are the corresponding phases. Here for simplicity we assume the DOP's of the pilot carrier and the message signal are preserved along the transmission fiber. The degradation caused by DOP will be discussed latter. Since the output PSP's are independent of frequency to first-order and the frequency deviation between the two waves is small, in the following we take $\hat{b}_{ \pm}\left(w_{s}\right)=\hat{b}_{ \pm}\left(w_{c}\right)$. The output PSP's are functions of the details of fiber birefringence and the perturbations through the propagation path which are difficult to be explicitly expressed. However, they do preserve the unitary and orthogonal properties [5], written as

$$
\begin{aligned}
\left|\hat{b}_{ \pm}\right|^{2} & =1 \\
\hat{b}_{+} \cdot \hat{b}_{-}^{*} & =0 .
\end{aligned}
$$

At the receiving end, the combined wave $\vec{E}(t)=\vec{E}_{s}(t)+$ $\vec{E}_{c}(t)$ is directly incident on a photodetector to produce photocurrent as

$$
\begin{aligned}
i(t) & =\frac{R}{2}|\vec{E}(t)|^{2} \\
& =\frac{R}{2}\left(\left|\vec{E}_{s}\right|^{2}+\left|\vec{E}_{c}\right|^{2}+\vec{E}_{s} \cdot \vec{E}_{c}^{*}+\vec{E}_{c} \cdot \vec{E}_{c s}^{*}\right)
\end{aligned}
$$

where $R$ is the photodetector responsivity and '*' denotes complex conjugate. Using the unitary and orthogonal properties of $b_{ \pm}$and neglecting the dc terms, the IF current is obtained as

$$
\begin{array}{r}
i_{\mathrm{IF}}(t)=R A_{s} A_{c} e^{-\alpha L}\left[c_{+}^{2} \cos \left(w_{\mathrm{IF}} t+m(t)+\Delta \phi+\theta_{+}\right)+c_{-}^{2}\right. \\
\left.\cdot \cos \left(w_{\mathrm{IF}} t+m\left(t-\tau_{p}\right)+\Delta \phi+\theta_{-}\right)\right]
\end{array}
$$

where $\theta_{ \pm}=\psi_{ \pm}\left(w_{s}\right)-\psi_{ \pm}\left(w_{c}\right)$. Also $w_{\mathrm{IF}}=w_{s}-$ $w_{c}$ is the intermediate frequency and $\Delta \phi=\phi_{s}-$ $\phi_{c}$.

The differential group delay $\tau_{p}$ which causes time delay between the information $m(t)$ and $m\left(t-\tau_{p}\right)$ will degrade the eye opening at decision level which had been reported in [8]. Here for simplicity we assume $\tau_{p}$ is small compare to a bit duration such that $m\left(t-\tau_{p}\right)$ is taken to be the same as $m(t)$ in the following analyses. We can reformulate $i_{\mathrm{IF}}(t)$ as

$$
i_{\mathrm{IF}}(t)=D_{1} \cdot R A_{s} A_{c} e^{-\alpha L} \cos \left(w_{\mathrm{IF}} t+m(t)+\Delta \phi+\xi\right)
$$

with

$$
\begin{aligned}
D_{1} & =\sqrt{c_{+}^{4}+2 c_{+}^{2} c_{-}^{2} \cos \Delta \theta+c_{-}^{4}} \\
\xi & =\theta_{+}+\tan ^{-1} \frac{c_{-}^{2} \sin \Delta \theta}{c_{+}^{2}+c_{-}^{2} \cos \Delta \theta} \\
\Delta \theta & =\theta_{-}-\theta_{+} .
\end{aligned}
$$

If no PMD represents the two linearly polarized waves will sustain linear polarization throughout the distribution fiber. In this case the IF signal can be easily obtained as

$$
i_{\mathrm{IF}}(t)=R A_{s} A_{c} e^{-\alpha L} \cos \left(w_{\mathrm{IF}} t+m(t)+\Delta \phi+\xi^{\prime}\right)
$$

where $\xi^{\prime}$ is a constant phase delay.

It is evident by observing (10) and (14) that the constant phases $\xi$ and $\xi^{\prime}$ are irrelevant to the IF signal. The degradation caused by PMD is solely determined by the factor $D_{1}$ which depends both on $c_{ \pm}$as well as $\Delta \theta$. It is easy to show

$$
0 \leq D_{1} \leq\left(c_{+}^{2}+c_{-}^{2}\right)^{2}=1
$$

which indicates that the PMD will in general degrade the IF signal amplitude. For the special case that only one PSP is excited $\left(c_{+}^{2}=1\right.$ and $c_{-}^{2}=0$, or vice versa), we have $D_{1}=1$. Also $D_{1}=1$ when $\Delta \theta=0$, is independent of $c_{ \pm}$. It is easy to show that the minimum value of $D_{1}$, corresponding to the worst case degradation, occurs when $c_{ \pm}^{2}=0.5$, i.e., the two PSP's are equally excited.

The factor $\Delta \theta$ can be estimated from the differential group delay between the two PSP's. Let the phase difference between the two PSP's be

$$
\eta(w)=\psi_{-}(w)-\psi_{+}(w)
$$

then

$$
\begin{aligned}
\Delta \theta & =\psi_{-}\left(w_{s}\right)-\psi_{-}\left(w_{c}\right)-\psi_{+}\left(w_{s}\right)+\psi_{+}\left(w_{c}\right) \\
& =\eta\left(w_{s}\right)-\eta\left(w_{c}\right) .
\end{aligned}
$$


The differential group delay between the two PSP's is related to $\eta(w)$ as [9]

$$
\tau_{p}=\frac{d \eta}{d w}
$$

Thus

$$
\Delta \theta=\int_{w_{c}}^{w_{s}} \tau_{p} d w
$$

From the measured results $\tau_{p}$ over a small frequency range (few gigahertzs) is essentially constant [9], therefore $\Delta \theta$ is approximately given as

$$
\Delta \theta \simeq \tau_{p} w_{\mathrm{IF}}
$$

Then

$$
D_{1}=\sqrt{c_{+}^{4}+2 c_{+}^{2} c_{-}^{2} \cos \left(\tau_{p} w_{\mathrm{IF}}\right)+c_{-}^{4}}
$$

and the worst-case degradation due to SOP mismatch $\left(c_{ \pm}^{2}=0.5\right)$ is

$$
D_{1}=\cos \left(\frac{\tau_{p} w_{\mathrm{IF}}}{2}\right)
$$

\section{B. Effect of DOP}

Even though a completely polarized wave is launched at the transmitting end, the received wave is in general partially polarized due to the presence of PMD [6]. The field of a partially polarized wave can be formulated as

$$
\vec{E}=\vec{E}_{\mathrm{pol}}+\vec{E}_{\text {dep }}
$$

where $\vec{E}_{\text {pol }}$ and $\vec{E}_{\text {dep }}$ are the completely polarized and depolarized components of $\vec{E}$, respectively, and they have uncorrelated phases $[10]$. The total intensity of the wave is given as

$$
I_{\mathrm{tot}}=I_{\mathrm{pol}}+I_{\mathrm{dep}}
$$

where $I_{\mathrm{pol}}$ and $I_{\mathrm{dep}}$ are the intensities of the completely polarized and depolarized components, respectively. The completely depolarized component has the same property as natural light that its intensity in any direction perpendicular to the propagation direction is the same. The DOP of $\vec{E}$ is defined as

$$
P=\frac{I_{\mathrm{pol}}}{I_{\mathrm{tot}}}
$$

which is the ratio of the intensity of the completely polarized component to total intensity. The intensity of the completely depolarized component is equal to

$$
I_{\mathrm{dep}}=I_{\mathrm{tot}}(1-P) \text {. }
$$

Let the incident pilot carrier and message signal be completely polarized waves with unit DOP. The presence of PMD will degrade the DOP as the waves propagate along the fiber, and therefore the received waves are in general partially polarized waves. It is shown that a partially polarized wave can be regarded as the sum of a completely polarized wave and a completely depolarized wave which have uncorrelated phases [10]. It is the completely polarized components of the two waves that contribute to the IF signal whereas the depolarized components, which are completely randomly polarized, will introduce additional noise to the IF signal. At the plane perpendicular to the propagation direction, the received fields can be expressed by the completely polarized and depolarized components. To simplify the analysis we assume, without loss of generality, the completely polarized components of the waves be linearly polarized and point along the same direction defined by a unit vector $\vec{x}$. Note here the completely polarized components of the two waves have the same SOP while the affect of SOP mismatch, which had been discussed previously is neglected. The received waves are expressed as

$$
\begin{aligned}
\vec{E}_{s}(t)=\sqrt{P_{s}} A_{s} e^{-\alpha \frac{L}{2}} e^{j\left[w_{s} t+m(t)+\phi_{s}\right]} \vec{x}+\sqrt{\frac{1-P_{s}}{2}} \\
\cdot A_{s} e^{-\alpha \frac{L}{2}} e^{j\left[w_{s} t+m(t)+\phi_{s}\right]}\left[e^{j \phi_{s x}} \vec{x}+e^{j \phi_{s y}} \vec{y}\right]
\end{aligned}
$$

$\vec{E}_{c}(t)=\sqrt{P_{c}} A_{c} e^{-\alpha \frac{L}{2}} e^{j\left(w_{c} t+\phi_{c}\right)} \vec{x}+\sqrt{\frac{1-P_{c}}{2}}$

$$
\cdot A_{c} e^{-\alpha \frac{L}{2}} e^{j\left(w_{c} t+\phi_{c}\right)}\left[e^{j \phi_{c x}} \vec{x}+e^{j \phi_{c y}} \vec{y}\right]
$$

where $P_{s}$ and $P_{c}$ denote the DOP's of the message signal and pilot carrier, respectively. The first terms on the right-hand side of the above two equations denote the completely polarized components of the waves and the second terms denote the depolarized components. The depolarized components are expressed by two orthogonal unit vectors $\vec{x}$ and $\vec{y}$ with uncorrelated phases $\phi_{s, c x}$ and $\phi_{s, c y}$. The phases of the depolarized components are also uncorrelated with those of the polarized components. Because $\phi_{s x}$ and $\phi_{s y}$ are uncorrelated, it is easy to show that the intensity of the depolarized component of $\vec{E}_{s}$ along any direction at the plane perpendicular to the propagation direction is the same. The intensity of the depolarized component of $\vec{E}_{s}$ is the sum of the intensities along $\vec{x}$ and $\vec{y}$ directions, obtained as

$$
I_{\mathrm{dep}}=\frac{1}{2}\left(1-P_{s}\right) A_{s}^{2} e^{-\alpha L}
$$

and the total intensity of $\vec{E}_{s}(t)$ is readily given as

$$
I_{\mathrm{tot}}=\frac{1}{2} P_{s} A_{s}^{2} e^{-\alpha L}+\frac{1}{2}\left(1-P_{s}\right) A_{s}^{2} e^{-\alpha L}=\frac{1}{2} A_{s}^{2} e^{-\alpha L} \text {. }
$$

Hence (26) is satisfied accordingly. Therefore (27) can express the partially polarized wave $\vec{E}_{s}(t)$. Similar argument is applicable to $\vec{E}_{c}(t)$.

From (27) and (28) we can obtain the IF current as

$$
\begin{aligned}
i_{\mathrm{IF}}(t)=R A_{s} A_{c} e^{-\alpha L} \\
\cdot\left[\sqrt{P_{s} P_{c}} \cos \left(w_{\mathrm{IF}} t+m(t)+\Delta \phi\right)+k_{s}\right. \\
\cdot \cdot \cos \left(w_{\mathrm{IF}} t+m(t)+\Delta \phi+\phi_{s x}\right)+k_{c} \\
\left.\cdot \cos \left(w_{\mathrm{IF}} t+m(t)+\Delta \phi-\phi_{c x}\right)\right]
\end{aligned}
$$


where

$$
\begin{aligned}
& k_{s}=\sqrt{\frac{\left(1-P_{s}\right) P_{c}}{2}} \\
& k_{c}=\sqrt{\frac{P_{s}\left(1-P_{c}\right)}{2}}
\end{aligned}
$$

and $w_{\mathrm{IF}}$ and $\Delta \phi$ are given as before. Here the terms result from the cross product of the two depolarized components, which should be much smaller than the other terms for reasonable system performance, are neglected. Note that in addition to the cross product of the completely polarized components, two noises resulting from the cross product of the polarized and depolarized components, are present in the IF signal. Consequently, the affect of DOP on the IF signal is twofold. First, it degrades the signal amplitude by a factor $\sqrt{P_{s} \bar{P}_{c}}$. Second, it results in IF noises due to the introduction of depolarized components. Because $\phi_{s, c x}$ are uncorrelated to $\phi_{s, c}$, they can be taken as random phases which are uniformly distributed within $[0,2 \pi]$. We can reformulate $i_{\mathrm{IF}}(t)$ as

$$
i_{\mathrm{IF}}(t)=R A_{s} A_{c} e^{-\alpha L} \cdot\left[Z \cos \left(w_{\mathrm{IF}} t+m(t)+\Delta \phi+\Lambda\right)\right]
$$

where (see equations (35) and (36) at bottom of page).

The above equations indicate that the noises induced by the depolarized components result in amplitude as well as phase noises on the IF signal. For $\sqrt{P_{s} \bar{P}_{c}} \gg k_{s, c}$, which is valid for reasonable performance, $Z$ and $\Lambda$ can be approximated as [11]

$$
\begin{aligned}
& Z \simeq \sqrt{P_{s} P_{c}}+k_{s} \cos \phi_{s x}+k_{c} \cos \phi_{c x} \\
& \Lambda \simeq \frac{k_{s} \sin \phi_{s x}-k_{c} \sin \phi_{c x}}{\sqrt{P_{s} P_{c}}} .
\end{aligned}
$$

It is clear that the amplitude and phase noises are nonGaussian noises because the distributions of $\cos \Phi_{s, c}$ are nonGaussian. Because of the non-Gaussian noises, it is difficult to compare these noises with the other receiver noises which are usually Gaussian distributed. The problem becomes even more complicated because the affect of these noises on system performance should be evaluated after nonlinear demodulation and baseband filtering. Instead of getting into these details, here for simplicity we calculate their variances and define a polarized signal to depolarized noise ratio at the IF stage. Using the fact that $\phi_{s, c x}$ are uniformly distributed within $[0,2 \pi]$, the variances of $Z$ and $\Lambda$ are obtained as

$$
\begin{aligned}
\sigma_{Z}^{2} & =\frac{1}{4}\left(P_{s}+P_{c}-2 P_{s} P_{c}\right) \\
\sigma_{\Lambda}^{2} & =\frac{P_{s}+P_{c}-2 P_{s} P_{c}}{4 P_{s} P_{c}} .
\end{aligned}
$$

We further define the polarized signal to depolarized noise ratio for the amplitude noise, which is the polarized signal power to noise variance, is obtained as

$$
K=10 \log \frac{P_{s} P_{c}}{\sigma_{Z}^{2}}=10 \log \frac{4 P_{s} P_{c}}{P_{s}+P_{c}-2 P_{s} P_{c}} .
$$

\section{Discussion}

We have shown that the IF signal degradation results from the PMD induced SOP mismatch and DOP degradation. The affect caused by SOP mismatch is the amplitude degradation of the IF signal whereas DOP causes IF signal amplitude degradation as well as additional IF noises.

A statistical treatment reveals that the differential group delay $\tau_{p}$ is proportional to the square root of fiber length if the length is far larger than the correlation length of perturbations [12]. Let $\tau_{u}$ denote the differential group delay per unit square root fiber length. Then

$$
\tau_{p}=\tau_{u} \cdot \sqrt{L} .
$$

For a completely polarized wave incident on the fiber, $P=1$, the DOP at the receiving end is given as [6]

$$
P=\sqrt{1-4 c_{+}^{2} c_{-}^{2}\left(1-|\gamma|^{2}\right)}
$$

where $c_{ \pm}$are again the expansion coefficients and the parameter $\gamma$ is

$$
\gamma=\frac{\int_{0}^{\infty} V(w) e^{j\left(w-w_{0}\right) \tau_{p}} d w}{\int_{0}^{\infty} V(w) d w}
$$

where $V(w)$ is the power spectrum of the light source and $w_{0}$ is the center frequency. From (43) we see that the DOP is preserved if only one PSP is excited and the minimum DOP occurs when both PSP's are equally excited $\left(c_{ \pm}^{2}=0.5\right)$. The minimum DOP is

$$
P=|\gamma|
$$

where $\gamma$ depends on the source spectrum $V(w)$ and the differential group delay $\tau_{p}$.

Under the worst-case condition that both PSP's are equally excited, from (22) and (31) the amplitude degradation of the IF signal is written as

$$
D=\cos \frac{\tau_{u} \sqrt{L} w_{\mathrm{IF}}}{2} \cdot \sqrt{\left|\gamma_{c} \gamma_{s}\right|}
$$

where the first-term on the right-hand side of (46) denotes the amplitude degradation caused by SOP mismatch and the

$$
\begin{aligned}
& Z=\sqrt{\left(\sqrt{P_{s} P_{c}}+k_{s} \cos \phi_{s x}+k_{c} \cos \phi_{c x}\right)^{2}+\left(k_{s} \sin \phi_{s x}-k_{c} \sin \phi_{c x}\right)^{2}} \\
& \Lambda=\tan ^{-1} \frac{k_{s} \sin \phi_{s x}-k_{c} \sin \phi_{c x}}{\sqrt{P_{s} P_{c}+k_{s} \cos \phi_{s x}+k_{c} \cos \phi_{c x}}} .
\end{aligned}
$$




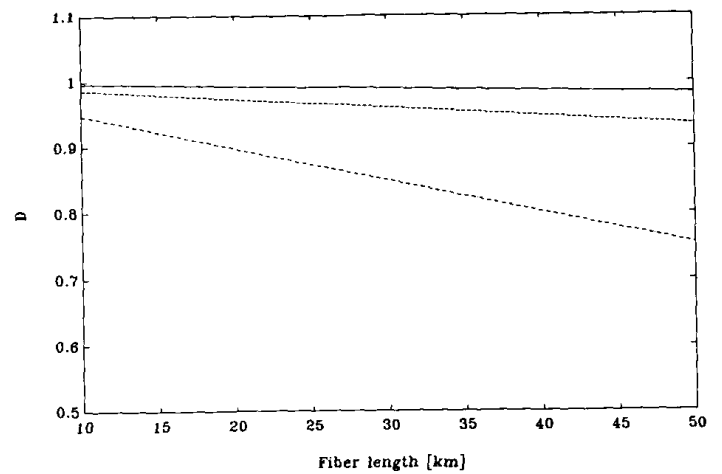

Fig. 1. The amplitude degradation factor versus fiber length. $w_{\mathrm{IF}}=$ $2 \pi \times 8 \mathrm{GHz}, \Delta w_{c}=2 \pi \times 40 \mathrm{MHz}, T=1 \mathrm{~ns}$. Solid line: $\tau_{u}=1 \mathrm{ps} / \sqrt{\mathrm{km}}$, dash line: $\tau_{u}=2 \mathrm{ps} / \sqrt{\mathrm{km}}$, dash-dotted line: $\tau_{u}=4 \mathrm{ps} / \sqrt{\mathrm{km}}$.

second term is due to DOP degradation. And the polarized signal to depolarized noise ratio is

$$
K=10 \log \frac{4\left|\gamma_{s} \gamma_{c}\right|}{\left|\gamma_{s}\right|+\left|\gamma_{c}\right|-2\left|\gamma_{s} \gamma_{c}\right|}
$$

and the variance of the phase noise $\Lambda$ is

$$
\sigma_{\Lambda}^{2}=\frac{\left|\gamma_{s}\right|+\left|\gamma_{c}\right|-2\left|\gamma_{s} \gamma_{c}\right|}{4\left|\gamma_{s} \gamma_{c}\right|}
$$

Let the pilot carrier have a Lorentzian spectral distribution with linewidth $\Delta w_{c}$, then the parameter $\gamma$ for the pilot carrier is given as [6]

$$
\gamma_{c}=\exp \left(-\Delta w_{c} \tau_{p}\right)
$$

The spectral distribution of the message signal depends on the light source and the modulation which varies widely. In the following we consider a coherent minimum shift keying (MSK) signal with power spectral density [13]:

$$
V(w)=\left[\frac{\cos \left(w-w_{0}\right) T}{1-\frac{4}{\pi^{2}}\left(w-w_{0}\right)^{2} T^{2}}\right]^{2}
$$

where $T$ is the bit duration and for simplicity we assume the source linewidth be small compared with the bit rate so that the spectrum mainly determined by the MSK modulation. The formulation given here can be applied to other modulation formats like PSK or FSK which have different power spectral densities and may result in different DOP degradation. With $V(w)$ we can calculate the parameter $\gamma_{s}$ of the message signal and then obtain its DOP.

The amplitude degradation factor $D$ as a function of fiber length is shown in Fig. 1 for $w_{\mathrm{IF}}=2 \pi \times 8 \mathrm{GHz}$. The degradation is little if $\tau_{u}$ is small but becomes significant when $\tau_{u}$ is large. We further note that $D$ decreases about linearly with fiber length. To understand the relative contribution of SOP and DOP to the degradation factor $D$, we show in Fig. 2 the magnitude of $D_{1}=\cos \left(\tau_{u} \sqrt{L} w_{\mathrm{IF}} / 2\right)$ and $D_{2}=$ $\sqrt{\left|\gamma_{c} \gamma_{s}\right|}$, which are the amplitude degradation factors due to SOP mismatch and DOP degradation, respectively, and the combined degradation factor $D=D_{1} D_{2}$ is also plotted

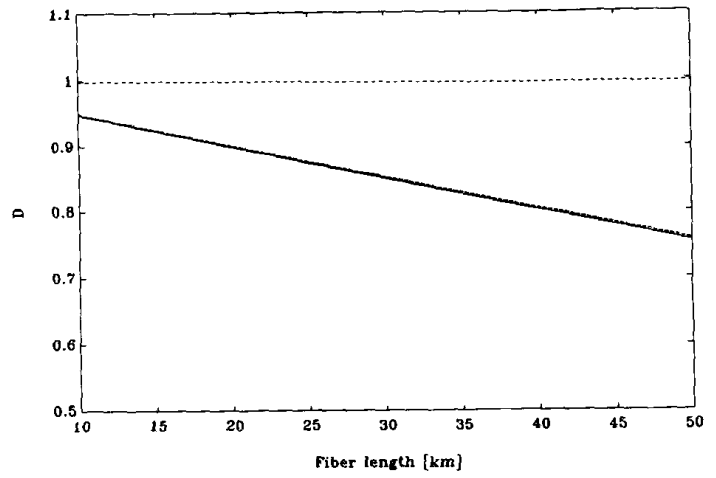

Fig. 2. The amplitude degradation factors versus fiber length for $\tau_{u}=$ $4 \mathrm{ps} / \sqrt{\mathrm{km}}, w_{\mathrm{IF}}=2 \pi \times 8 \mathrm{GHz}$. Dash line: $D_{1}=\cos \left(\tau_{u} \sqrt{L} w_{\mathrm{IF}} / 2\right)$, dash-dotted: $D_{2}=\sqrt{\left|\gamma_{s} \gamma_{c}\right|}$, solid line: $D=D_{1} D_{2}$. Note that $D_{1}$ nearly coincides with $D$.

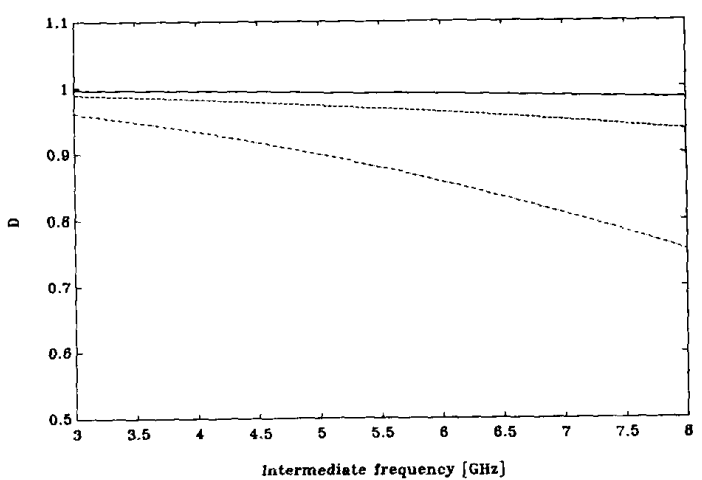

Fig. 3. The amplitude degradation factor versus intermediate frequency for $L=50 \mathrm{~km}$. Solid line: $\tau_{u}=1 \mathrm{ps} / \sqrt{\mathrm{km}}$, dash line: $\tau_{u}=2 \mathrm{ps} / \sqrt{\mathrm{km}}$, dash-dotted line: $\tau_{u}=4 \mathrm{ps} / \sqrt{\mathrm{km}}$.

for $\tau_{u}=4 p s / \sqrt{\mathrm{km}}$. The figure reveals that the amplitude degradation is mainly due to $D_{1}$ while $D_{2}$ contributes little, which means that in this case the amplitude degradation is majorly caused by SOP mismatch but not DOP degradation. Fig. 3 illustrates the relationship between $D$ and the intermediate frequency for $L=50 \mathrm{~km}$. $D$ decreases as the intermediate frequency increases and significant degradation occurs for large $\tau_{u}$. We further show in Fig. 4 the polarized signal to depolarized noise ratio as a function of fiber length. Again the ratio decreases with fiber length because of DOP degradation. The variance of $\Lambda$ as a function of fiber length is shown in Fig. 5. It is seen that $\sigma_{\Lambda}^{2}$ increases with fiber length and the unit differential group delay. In the examples we see that $K$ is large and $\sigma_{\Lambda}^{2}$ is small, consequently the affect of the depolarized noise is expected to be insignificant. However, if there is severe DOP degradation along the transmission fiber, the affect of the depolarized noises could be pronounced which may significantly degrade system performance. In this case more careful study is necessary to evaluate the real impact of the depolarized noises on the system performance after demodulation and baseband filtering. 


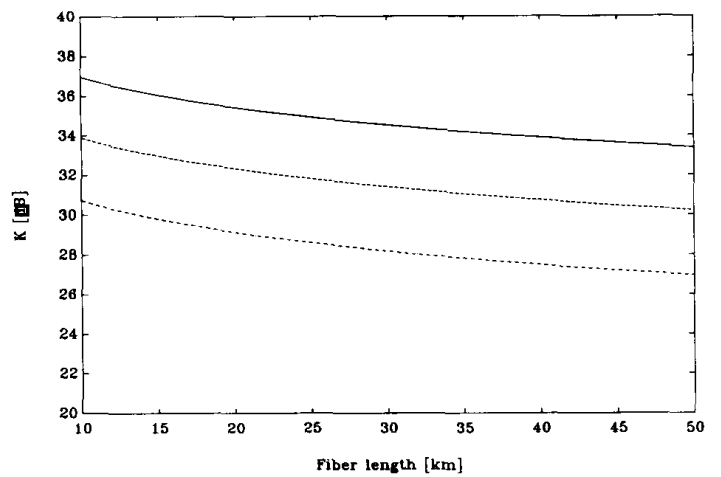

Fig. 4. $K$ as a function of fiber length for $w_{\mathrm{IF}}=2 \pi \times 8 \mathrm{GHz}$. Solid line: $\tau_{u}=1 \mathrm{ps} / \sqrt{\mathrm{km}}$, dash line: $\tau_{u}=2 \mathrm{ps} / \sqrt{\mathrm{km}}$, dash-dotted line: $\tau_{u}=4 \mathrm{ps} / \sqrt{\mathrm{km}}$

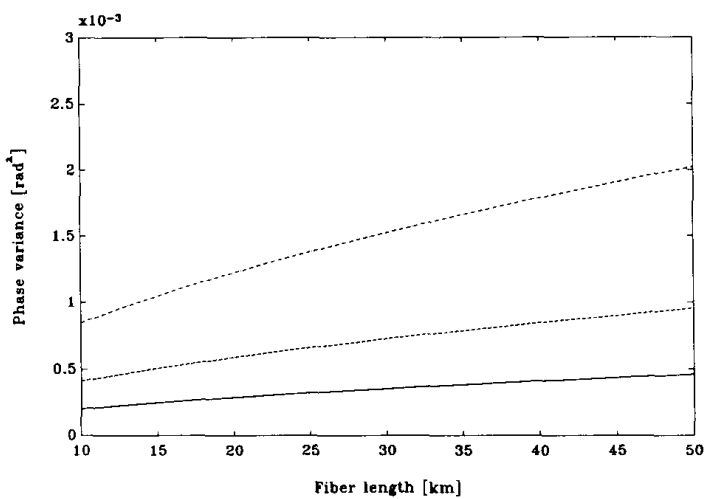

Fig. 5. $\sigma_{\Lambda}^{2}$ as a function of fiber length for $w_{\mathrm{IF}}=2 \pi \times 8 \mathrm{GHz}$. Solid line: $\tau_{u}=1 \mathrm{ps} / \sqrt{\mathrm{km}}$, dash line: $\tau_{u}=2 \mathrm{ps} / \sqrt{\mathrm{km}}$, dash-dotted line $\tau_{u}=4 \mathrm{ps} / \sqrt{\mathrm{km}}$

\section{CONCLUSION}

In summary we have evaluated the performance degradation due to polarization mode dispersion in a coherent optical system with pilot carrier. Both the PMD induced SOP and DOP changes are analyzed. The affect of SOP causes IF signal amplitude degradation which depends on the fiber length, the unit differential group delay, and the intermediate frequency. On the other hand, the affect of DOP not only causes IF signal amplitude degradation by also introduces additional noises due to the depolarized components. The noises are non-Gaussian which differ from usual receiver noises. We provide analytical expressions to evaluate the performance degradation. The results show that there is no performance degradation if only one PSP is excited and worst case degradation occurs when both PSP's are equally excited. The degradation is little if the differential group delay between the PSP's is small and becomes significant if the delay is large.

\section{REFERENCES}

[1] J.A. Arnaud, "Enhancement of optical receiver sensitivity by amplification of the carrier," IEEE J. Quantum Electron., vol. QE-4, no. 11, pp. 893-899, Nov. 1968.

[2] C. G. Atkins, D. Cotter, D. W. Smith, and R. Wyatt, "Application of Brillouin amplification in coherent optical transmission" IEE Electron. Lett., vol. 22, no. 10, pp. 5656-558, May 1986.

[3] M.S. Kao and J. Wu, "Coherent subcarrier multiplexed star distribution system using single local oscillator" IEE Electron. Lett., vol. 26, pp. $1680-1682$, Sept. 1990.

[4] R. Gross, W. Rideout, R. Olshansky, and G. R. Joyce, "Heterodyne video distribution systems sharing transmitter and local oscillator lasers," $J$. Lightwave Technol., vol. 9, pp. 524-530, Apr. 1991.

[5] C. D. Poole and R. E. Wagner, "Phenomenological approach to polarization dispersion in long single-mode fibers," IEE Electron. Lett., vol. 22, no. 19, pp. 1029-1030; Sept. 1986.

[6] J. Sakai, S. Machida, and T. Kimura, "Degree of polarization in anisotropic single-mode optical fibers: Theory,"IEEE J. Quantum Electron., vol. QE-8, no. 4, pp. 488-495, Apr. 1982.

[7] M.S. Kao and J. Wu, "Performance degradation due to polarization mode dispersion in a coherent optical system with pilot carrier" IEEE Photon. Technol. Lett., vol. 3, no. 3, pp. 265-267, Mar. 1991.

[8] R.E. Wagner and A.F. Elrefaie, "Polarization dispersion limitations in lightwave systems," OFC'88, 1988, paper Tul6.

[9] C.D. Poole, N.S. Bergano, R.E. Wagner, and H. J. Schulte, "Polarization dispersion and principal states in a 147-km undersea lightwave cable," J. Lightwave Technol., vol. 6, no. 7, pp. 1185-1190, July 1988

[10] M. Born and E. Wolf, Principles of Optics. New York: Pergamon, 1980.

[11] R.E. Ziemer and W. H. Tranter, Principles of Communications Boston: Houghton Mifflin, 1985, ch. 6.

[12] F. Curti, B. Daino, G. D. Marchis, and F. Matera, "Statistical treatment of the evolution of the principal states of polarization in single-mode fibers," J. Lightwave Technol., vol. 8, no. 8, pp. 1162-1166, Aug. 1990.

[13] J. G. Proakis, Digital Communications. New York: McGraw-Hill, 1989.

Minh-Seng Kao, photograph and biography not available at the time of publication

Jingshown Wu, photograph and biography not available at the time of publication. 